\title{
A Comparison of Three-Dimensional Simulations of Traveling-Wave Tube Cold-Test Characteristics Using CST MICROWAVE STUDIO and MAFIA
}

\author{
C. T. Chevalier ${ }^{1}$ Christine.Chevalier@grc.nasa.gov, K. A. Herrmann ${ }^{2}$, C. L. Kory ${ }^{1}$, J. D. \\ Wilson ${ }^{2}$, A. W. Cross ${ }^{2}$
}

Analex Corporation ${ }^{1} /$ NASA Glenn Research Center ${ }^{2}$

21000 Brookpark Road, MS 54-5

Cleveland, $\mathrm{OH} 44135$

Phone: 216-433-6082

Fax: 216-433-8705

Subject Category: Technologies - Analysis and Computer Modeling

Previously, it was shown that MAFIA (solutions of Maxwell's equations by the Finite Integration Algorithm) [1,2], a three-dimensional simulation code, can be used to produce accurate cold-test characteristics including frequency-phase dispersion, interaction impedance, and attenuation for traveling-wave tube (TWT) slow-wave structures [3]. In an effort to improve user-friendliness and simulation time, a model was developed [4] to compute the cold-test parameters using the electromagnetic field simulation software package CST MICROWAVE STUDIO (MWS) [2]. Cold-test parameters were calculated for several slow-wave circuits including a ferruled coupled-cavity, a folded waveguide, and a novel finned-ladder circuit using both MWS and MAFIA. Comparisons indicate that MWS provides more accurate cold-test data with significantly reduced simulation times.

Both MAFIA and MWS are based on the finite integration (FI) method; however, MWS has several advantages over MAFIA. First, it has a Windows based interface for PC operation, making it very user-friendly, whereas MAFIA is UNIX based. MWS uses a new Perfect Boundary Approximation (PBA), which increases the accuracy of the simulations by avoiding stair step approximations associated with MAFIA's representation of structures. Finally, MWS includes a Visual Basic for Applications (VBA) compatible macro language that enables the simulation process to be automated and allows for the optimization of user-defined goal functions, such as interaction impedance. One present disadvantage of MWS is a lack of periodic boundary conditions, which requires the user to model several cavities of the slow-wave structure and to truncate each section with electric or magnetic boundaries in order to calculate the resonant frequencies at corresponding phase shifts per cavity [3]. However, periodic boundaries will be implemented into MWS in the future.

Cold-test simulations, including frequency-phase dispersion, interaction impedance, and attenuation, using MWS and MAFIA will be compared for three types of TWT slow-wave circuits: a ferruled coupled-cavity TWT circuit, a folded waveguide TWT circuit, and a novel finned-ladder TWT developed at NASA GRC [5]. For all cases, improvements in accuracy and dramatic savings in time were achieved. For example, the simulation time was 8.6 times faster for the finned-ladder circuit simulations with MWS on a PC compared to MAFIA on a Sun Ultra 80 Workstation. Figure 1 illustrates the user-friendly MWS graphical user interface (GUI) with a three-dimensional cutaway of three-cavities of the ferruled coupled-cavity circuit. The electric field is shown at a phase shift of $300^{\circ}$ per cavity, with the size and color of the arrows proportional to the magnitude of the field.

This is a preprint or reprint of a paper intended for presentation at a conference. Because changes may be made before formal publication, this is made available with the understanding that it will not be cited or reproduced without the permission of the author. 
Work supported by NASA's Computation Information and Communications Technology (CICT) program in Code $\mathrm{R}$.

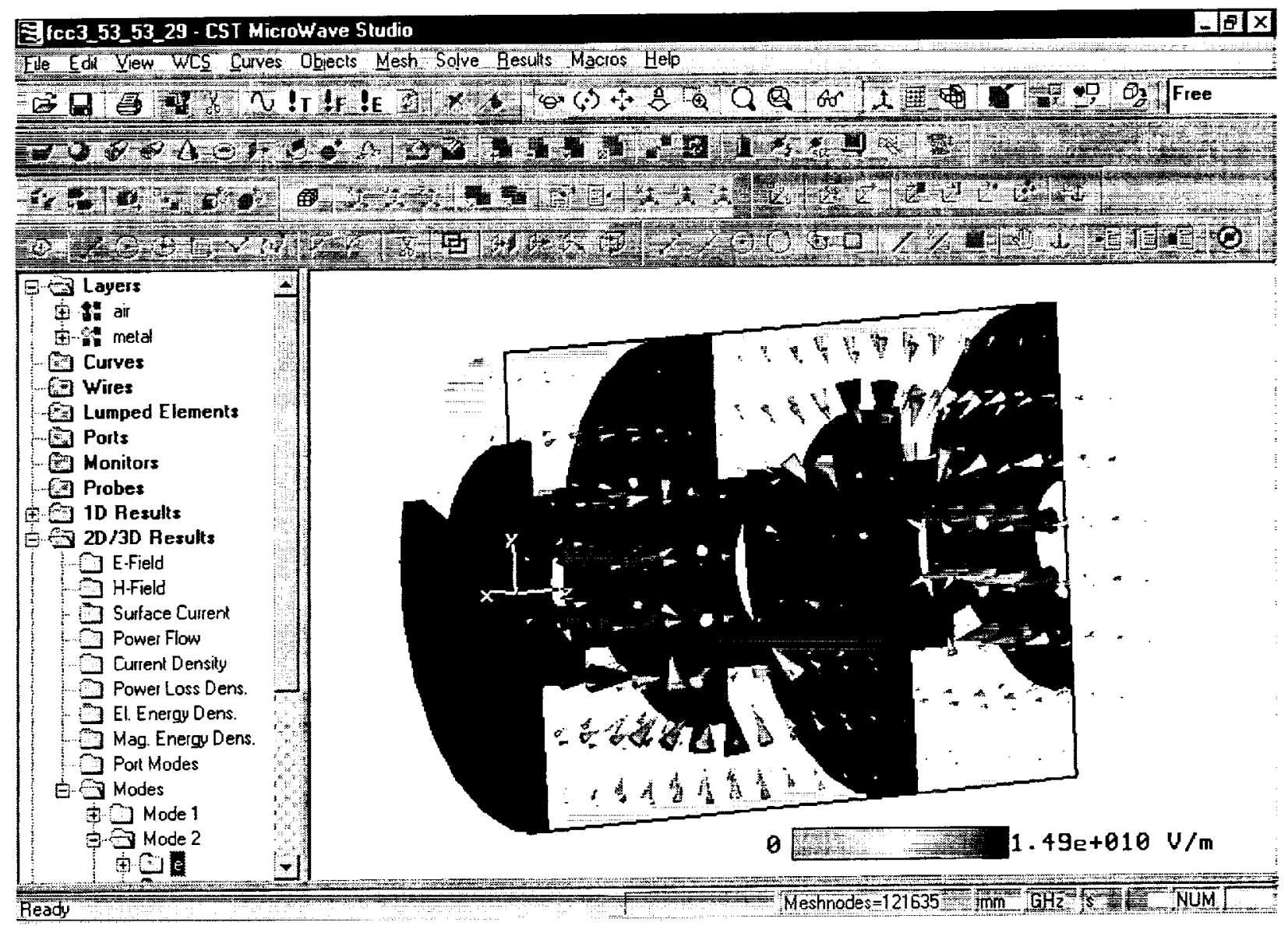

Figure 1.-MWS three-dimensional electric field plot for a TWT circuit. Size and color of three-dimensional arrows are proportional to magnitude of the field. Phase shift per cavity, $\beta \mathbf{L}, 300^{\circ}$.

$1 \mathrm{~T}$. Weiland, 1985, On the Unique Numerical Solution of Maxwellian Eigenvalue Problems in Three Dimensions, Part. Accel., vol. 17, pp. 227-242.

2 Computer Simulation Technology (CST), www.cst.de

3 Kory, C.L.; and Wilson, J.D.: Three-Dimensional Simulation of Traveling-Wave Tube Cold Test Characteristics Using MAFIA. NASA TP-3513, 1995.

4 Chevalier, C.T.; Herrmann, K.A.; Kory, C.L.; Wilson, J.D.; Cross, A.W.; and Santana, S.: Three-Dimensional Simulation of Traveling-Wave Tube Cold-Test Characteristics Using CST MICROWAVE STUDIO, NASA Technical Paper in preparation.

5 Patent filed. 